\title{
Electrostatic and thermal fluctuations are the source of magnetic fields in unmagnetized inhomogeneous plasmas
}

\author{
Hamid Saleem \\ National Centre for Physics (NCP), \\ Quaid-i-Azam University Campus,Islamabad, \\ Pakistan.
}

PACS:52.25Gj, 52.35. Fp, 52.50.Jm

19, May 2009

\begin{abstract}
It is pointed out that electron thermal fluctuations can couple with the ion acoustic mode in an inhomogeneous plasma to generate a low frequency ion time scale electromagnetic wave. This electromagnetic wave can become unstable if the temperature and density gradients are parallel to each other which can be the case in laser-plasmas similar to stellar cores. The comparisons of the present theoretical model with the previous investigations are also presented. The final result is applied to a classical laser induced plasma for illustration.
\end{abstract}

\section{Introduction}

A low frequency electromagnetic wave should be a normal mode of unmagnetized inhomogeneous plasmas as an intrinsic source of magnetic fields. The ion acoustic wave (IAW) is a very important mode because it exits in both unmag- 
netized and magnetized plasmas. It is a low frequency wave and light electrons are generally believed to act as inertia-less in its electrostatic field.

In fact the electron inertia can play a vital role in producing a low frequency electromagnetic wave. More than a decade ago [1], it was proposed that an electromagnetic wave having frequency near IAW is a normal mode of unmagnetized plasmas which can be responsible for magnetic field generation if it becomes unstable. In the derivation of its linear dispersion relation the electron inertia was not ignored while the displacement current was neglected. It was shown that the compressibility and vorticity can couple due to density inhomogeneity and hence a low frequency electromagnetic wave can be produced. Basically electrostatic IAW and magnetostatic mode [2] cooperate with each other to develop such a wave. The electron temperature perturbation was not taken into account and the steady state was assumed to be maintained by external mechanisms. The theory was applied to explain the magnetic field generation in laser plasmas. But the longitudinal and transverse characters of electric field decouple if the quasi-neutrality is used. Then both the high frequency and low frequency electromagnetic instabilities were also investigated in unmagnetized plasmas [3]. The interest in the investigation of low frequency magnetic fluctuations in unmagnetized plasmas was initiated after the first experimental observation of magnetic field generated in a laser induced plasma [4]. Then more experiments were performed $[5,6]$ on these lines. Several theoretical models were presented to explain the magnetic field generation in laser produced plasmas [7-13].

The magnetic electron drift vortex (MEDV) mode was proposed as a pure transverse linear mode which can exist because of the electron temperature fluctuations in unmagnetized inhomogeneous electron plasmas [13]. This mode can become unstable [14] if the equilibrium electron temperature gradient is parallel to the density gradient and it is maintained by external effects. 
Most of the mechanisms proposed to explain magnetic fluctuations in initially unmagnetized plasmas are based on electron magnetohydrodynamics (EMHD) which has been discussed in detail in Refs. $[15,16]$.

Recently [17], EMHD has been used to study long-lived and slowly propagating nonlinear whistler structures (NLWS), or whistler spheromaks (WSPS) observed in laboratory experiments $[18,19]$. In MEDV mode the divergence of electric field is assumed to be zero $\left(\nabla \cdot \mathbf{E}_{1}\right)=0$ while the divergence of electron velocity is non-zero $\left(\nabla \cdot \mathbf{v}_{e 1} \neq 0\right)$. Furthermore the electrons are inertia-less and ions are treated to be stationary. The frequency $\omega$ of the MEDV mode is assumed to lie in between the ion plasma frequency and electron plasma frequency i.e. $\omega_{p i}<<\omega<<\omega_{p e}$ where $\omega_{p j}=\left(\frac{4 \pi n_{0 j} e^{2}}{m_{j}}\right)$, for $\mathrm{j}=\mathrm{e}, \mathrm{i}$ and $\mathrm{c}$ is speed of light, while $\mathrm{k}$ is the wave vector. These restrictions and assumptions are indeed very strict.

It is important to find out some electromagnetic mode taking into account the ion dynamics so that the strict restrictions on the frequency and wavelength of the perturbation are relaxed. Then the only required condition on frequency should be $\omega<<\omega_{p e}, c k$. Furthermore, the electron temperature gradient is necessary for hydrostatic steady state $\nabla p_{e 0}=0$. In this case the temperature gradient becomes anti-parallel to density. But laser and astrophysical plasmas are open systems and many external mechanisms can maintain a study state with parallel density and temperature gradients. For example, in stellar cores, both the density and temperature increase towards centre of the star due to fusion and star is held intact because of gravity. Therefore both the parallel and anti-parallel gradients can be discussed. The electron thermal fluctuations can produce electromagnetic wave as was proposed many decades ago [13]. Several interesting works have appeared in literature on these lines (see for example review articles $[9,15,16])$. 
It is pointed out here that it is not necessary to assume a pure transverse perturbation in an electron plasma. Rather the perturbation can be partially transverse and partially longitudinal and hence the electron density perturbation may not be neglected. The frequency of such a wave in electron plasma turns out to be near $v_{t e} \kappa_{n}$ where $\left.v_{t e}=\left(\frac{T_{e}}{m_{e}}\right)\right)^{\frac{1}{2}}$ is the electron thermal speed and $\kappa_{n}=\left|\frac{1}{n_{0}} \frac{d n_{0}}{d x}\right|$ is the inverse of density gradient scale length $L_{n}=\frac{1}{\kappa_{n}}$. In the local approximation we need to have $\kappa_{n}<<k$. Furthermore the condition $\omega_{p i}^{2}<<v_{t e}^{2} k^{2}$ can be satisfied if $\frac{m_{e}}{m_{i}}<<\lambda_{D e}^{2} k_{y}^{2}$. Therefore, generally we may have $v_{t e} \kappa_{n} \lesssim \omega_{p i}$ and hence one cannot neglect ion dynamics. Moreover, $v_{t e} \kappa_{n} \simeq c_{s} k$ (where $c_{s}=\left(\frac{T_{e}}{m_{i}}\right)^{\frac{1}{2}}$ is ion sound speed) is also possible.

Therefore, it is expected that the electron thermal fluctuations can couple with IAW to produce stable and unstable low frequency electromagnetic waves in unmagnetized plasmas. We are interested mainly in finding out a quadratic dispersion relation for a low frequency electromagnetic wave to see a simplest physical picture. For a comparison and general interest, the previous theroretical models are also briefly discussed.

In the next section, the pure electron plasma is investigated including the description of MEDV mode. In section III, the coupling of IAW with magnetostatic mode due to inhomogeneity is briefly revised. In section IV a new electromagnetic mode which arises due to the coupling of IAW with thermal fluctuations is presented.In section $\mathrm{V}$ the result is applied to a case of classical plasma for illustration. Finally the physical assumptions and mathematical limits of the results are discussed.

\section{Electron Plasma}

Let us consider the electron plasma in the background of stationary ions. 
The set of equations in the linear limit can be written as,

$$
\begin{gathered}
m_{e} n_{0} \partial_{t} \mathbf{v}_{e 1}=-e n_{0} \mathbf{E}_{1}-\nabla p_{e 1} \\
\nabla \times \mathbf{B}_{1}=\frac{4 \pi}{c} \mathbf{J}_{1} \\
\mathbf{J}_{1}=-e n_{0} \mathbf{v}_{e 1} \\
\nabla \times \mathbf{E}_{1}=-\frac{1}{c} \partial_{t} \mathbf{B}_{1}
\end{gathered}
$$

Since

$$
p_{1}=n_{0} T_{e 1}
$$

therefore energy equation becomes,

$$
\frac{3}{2} n_{0} \partial_{t} T_{e 1}+\frac{3}{2} n_{0}\left(\mathbf{v}_{e 1} \cdot \nabla\right) T_{e 0}=-p_{0} \nabla \cdot \mathbf{v}_{e 1}
$$

First we describe the pure transverse MEDV mode.

Curl of (1) gives,

$$
\partial_{t}\left(\nabla \times \mathbf{v}_{e 1}\right)=\frac{e}{m_{e} c} \partial_{t} \mathbf{B}_{1}+\frac{1}{m_{e} n_{o}}\left(\nabla n_{0}\right) \times \nabla T_{e 1}
$$

Equations (2) and (3) yield,

$$
\mathbf{v}_{e 1}=-\frac{c}{4 \pi e n_{0}} \nabla \times \mathbf{B}_{1}
$$

and hence

$$
\nabla \times \mathbf{v}_{e 1}=\frac{c}{4 \pi e n_{0}} \nabla^{2} \mathbf{B}_{1}
$$

where $\nabla n_{0} \times\left(\nabla \times \mathbf{B}_{1}\right)=0$ due to the assumption $\mathbf{k} \perp \nabla n_{0} \perp \mathbf{B}_{1}$. Equation (8) predicts $\mathbf{E}_{1}=E_{1} \hat{\mathbf{x}}$ while $\nabla n_{0}=\hat{\mathbf{x}} \frac{d n_{0}}{d x}, \nabla=\left(0, i k_{y}, 0\right)$ and $\mathbf{B}_{1}=B_{1} \hat{\mathbf{z}}$ have been 
chosen.

Equation (6) and (8) yield,

$$
\left(1+\lambda_{e}^{2} k^{2}\right) \partial_{t} \mathbf{B}_{1}=-\frac{c}{e n_{0}}\left(\nabla n_{0} \times \nabla T_{e 1}\right)
$$

where $\lambda_{e}=\frac{c}{\omega}{ }_{p e}$. Equation (7) gives,

$$
\nabla \cdot \mathbf{v}_{e 1}=\frac{c}{4 \pi n_{0} e} \frac{\nabla n_{0}}{n_{0}} \cdot\left(\nabla \times \mathbf{B}_{1}\right)
$$

and therefore one obtains,

$$
T_{e 1}=\frac{2}{3} \frac{c}{4 \pi n_{0} e} k_{y} \kappa_{n} B_{1}
$$

Then (9) and (11) give the linear dispersion relation of MEDV mode as,

$$
\omega^{2}=\frac{2}{3} C_{0}\left(\frac{\kappa_{n}}{k_{y}}\right)^{2} v_{T e}^{2} k_{y}^{2}
$$

where $C_{0}=\frac{\lambda_{e}^{2} k^{2}}{1+\lambda_{e}^{2} k^{2}}$ and $v_{t e}=\left(T_{e} / m_{e}\right)^{\frac{1}{2}}$. If $\nabla T_{e 0} \neq 0$ is assumed, then (12) becomes,

$$
\omega^{2}=L_{0} \frac{\kappa_{n}}{k_{y}}\left[\frac{\left(\frac{2}{3} \kappa_{n}-\kappa_{T}\right)}{k_{y}}\right] v_{T e}^{2} k^{2}
$$

where $\kappa_{T}=\left|\frac{1}{T_{e 0}} \frac{d T_{e 0}}{d x}\right|$ and $\nabla T_{e 0}=+\mathbf{x} \frac{d T_{e 0}}{d x}$ has been used. If $\left(\frac{2}{3} \kappa_{n}-\kappa_{T}\right)<0$, then the mode becomes unstable[14].

It can be noticed that we have $\nabla \cdot \mathbf{E}_{1}=0$ and $\nabla \cdot \mathbf{v}_{e 1} \neq 0$ in the above treatment and it does not seem to be very convincing.

If we write (1) in $\mathrm{x}$ and $\mathrm{y}$ components, we can observe that the $\mathrm{y}$-component of $\mathbf{E}_{1}$ should not be considered as zero due to $\partial_{y} p_{e 1} \neq 0$ and hence the wave may be partially transverse and partially longitudinal. 
The curl of (2) yields a relation between $\mathbf{E}_{1 x}$ and $\mathbf{E}_{1 y}$,

$$
\mathbf{E}_{1 x}=-\frac{1}{a} \frac{\kappa_{n}}{k_{y}}\left(i E_{1 y}\right)
$$

where $a=\left(1+\lambda_{e}^{2} k_{y}^{2}\right)$.

Equation (5) becomes,

$$
W_{0}^{2} \frac{T_{e 1}}{T_{0}}=v_{t e}^{2} k_{y}^{2}\left(1-\frac{3}{2} \frac{\kappa_{T}^{2}}{k_{y}^{2}}\right) \frac{n_{e 1}}{n_{0}}-\frac{e}{m_{e}}\left(i k_{y} E_{1 y}+\frac{3}{2} k_{T} E_{1 x}\right)
$$

where $W_{0}^{2}=\frac{3}{2} \omega^{2}-v_{t e}^{2} k_{y}^{2}\left(1-\frac{3}{2} \frac{\kappa_{T} \kappa_{n}}{k_{y}^{2}}\right)$

The continuity equation yields,

$$
\begin{gathered}
L_{0}^{2} \frac{n_{e 1}}{n_{0}}=-\left(1+\frac{v_{t e}^{2} k_{y}^{2}}{W_{0}^{2}}\right)\left(i \frac{e}{m_{e}} k_{y} E_{1 y}\right) \\
-\frac{e}{m_{e}}\left(1+\frac{3}{2} \frac{\kappa_{T}}{\kappa_{n}} \frac{v_{t e}^{4} k_{y}^{4}}{W_{0}^{2}}\right) \kappa_{n} E_{1 x}
\end{gathered}
$$

where $L_{0}^{2}=\left\{\omega^{2}-v_{t e}^{2} k_{y}^{2}-\frac{v_{t e}^{4} k_{y}^{2}}{W_{0}^{2}}\left(1-\frac{3}{2} \frac{\kappa_{T}^{2}}{k_{y}^{2}}\right)\right\}$. The Poisson equation

$$
\nabla \cdot \mathbf{E}_{1}=-4 \pi e\left(\frac{n_{e 1}}{n_{0}}\right)
$$

can be written as,

$$
\begin{gathered}
\left\{\left[\frac{3}{2} \omega^{4}-v_{t e}^{2} k_{y}^{2}\left(\frac{3}{2}+G_{n}\right) \omega^{2}-\frac{3}{2} v_{t e}^{4} k_{y}^{4}\left(g_{T}^{2}-g_{n T}^{2}\right)\right]-\omega_{p e}^{2}\left[\frac{3}{2} \omega^{2}+\frac{3}{2} v_{t e}^{2} k_{y}^{2} g_{n T}^{2}\right]\right\} i E_{1 y} \\
=\omega_{p e}^{2}\left[\frac{3}{2} \omega^{2}-v_{t e}^{2} k_{y}^{2}\left(1-\frac{3}{2} g_{n T}^{2}-\frac{3}{2} \frac{\kappa_{T}}{\kappa_{n}}\right)\right] \frac{\kappa_{n}}{k_{y}} i E_{1 x}
\end{gathered}
$$

where $G_{n}=\left(1-\frac{3}{2} g_{n T}^{2}\right), g_{n T}^{2}=\frac{\kappa_{n} \kappa_{T}}{k_{y}^{2}}$ and $g_{T}^{2}=\kappa_{T}^{2} / k_{y}^{2}$.

Equations (14) and (18) yield a linear dispersion relation in the limit $\omega^{2}<<\omega_{p e}^{2}$ 
as,

$$
\omega^{2} \simeq \frac{2}{3 H_{0}}\left[-v_{t e}^{2} k_{y}^{2}\left(\frac{\kappa_{n}^{2}}{k_{y}^{2}}+\frac{3}{2} \lambda_{e}^{2} k_{y}^{2} g_{n T}^{2}\right)+\frac{3}{2} a \lambda_{D e}^{2} k_{y}^{2}\left(g_{T}^{2}-g_{n T}^{2}\right)\right]
$$

where $H_{0}=\left\{\left(1+\lambda_{D e}^{2} k_{y}^{2}\right) a-\frac{\kappa_{n}^{2}}{k_{y}^{2}}+a \lambda_{D e}^{2} k_{y}^{2} G_{n}\right\}$ and $\lambda_{D e}^{2}=\left(\frac{T_{e}}{4 \pi n_{0} e^{2}}\right)$. The last term on right hand side of (19) is smaller because $\lambda_{D e}^{2} k_{y}^{2}<1$ and due to local approximation we have $\kappa_{n}, \kappa_{T}<<k_{y}$. Therefore (19) can be simplified as,

$$
\omega^{2}=-\frac{2}{3 H_{0}} v_{t e}^{2} \kappa_{n}^{2}\left(1+\frac{3}{2} \lambda_{e}^{2} k_{y}^{2} \frac{\kappa_{T}}{\kappa_{n}}\right)
$$

which predicts a purely growing electromagnetic mode.

The dispersion relation (13) can not be retrieved from (20) because we have evaluation $\nabla \cdot \mathbf{v}_{1 e}$ from equation of motion and in the derivation of (13), the velocity $\mathbf{v}_{e 1}$ is evaluated using Ampere's law for pure transverse wave. The frequency $\omega$ in both the dispersion relations is of the order of $v_{t e}^{2} \kappa_{n}^{2}$ or smaller. The important point to note is that

$$
\omega^{2} \sim\left(\frac{\kappa_{n}}{k_{y}}\right)^{2}\left(v_{t e}^{2} k_{y}^{2}\right)=\left(\frac{\kappa_{n}}{k_{y}}\right)^{2}\left(\lambda_{D e}^{2} k_{y}^{2}\right) \omega_{p e}^{2}
$$

and $\lambda_{D e}^{2} k_{y}^{2}<<1$ has been assumed to ignore electron plasma wave. Therefore,

even for $\frac{m_{e}}{m_{i}}<\left(\frac{\kappa_{n}}{k_{y}}\right)^{2}$, the frequency in (21) can satisfy the relation $\omega \lesssim \omega_{p i}$ or even $\omega$ can be of the order of IAW frequency $c_{s} k_{y}$.

Therefore, the partially transverse and partially longitudinal electron wave of equation (20) may couple with ion acoustic mode.

\section{IAW and Magnetostatic Mode}

Here we shall show that transverse magnetostatic mode [2] which is obtained in the limit $\omega^{2}<<\omega_{p e}^{2}$ can couple with IAW in a nonuniform plasma [1]. Therefore, both ions and electrons are considered to be dynamic. The ions are assumed to 
be cold for simplicity and therefore equation of motion becomes,

$$
\partial_{t} \mathbf{v}_{i 1}=\frac{e}{m_{i}} \mathbf{E}_{1}
$$

The continuity equation yield,

$$
\frac{n_{i 1}}{n_{0}}=\frac{e}{m_{i} \omega^{2}}\left(\kappa_{n} E_{1 x}+i k_{y} E_{1 y}\right)
$$

If quasi-neutrality is used then transverse component $E_{1 x}$ and longitudinal component $E_{1 y}$ become uncoupled. Therefore, we assume $\lambda_{D e}^{2} k_{y}^{2} \neq 0$ and use Poisson equation which in the limit $\omega^{2}<<\omega_{p e}^{2}$ becomes,

$\left[-v_{t e}^{2} k_{y}^{2} \omega^{2}-\omega_{p i}^{2}\left(\omega^{2}-v_{t e}^{2} k_{y}^{2}\right)-\omega_{p e}^{2} \omega^{2}\right] i k_{y} E_{1 y} \simeq\left[\omega_{p i}^{2}\left(\omega^{2}-v_{t e}^{2} k_{y}^{2}\right)+\omega_{p e}^{2} \omega^{2}\right] \kappa_{n} E_{1 x}$

Note that the term $v_{t e}^{2} k_{y}^{2}$ is not ignored compared to $\omega_{p e}^{2}$ to couple $E_{1 x}$ and $E_{1 y}$. Equations (14) and (24) give a linear dispersion relation as,

$$
\omega^{2}=\frac{c_{s}^{2} k_{y}^{2}\left(a-\frac{\kappa_{n}^{2}}{k_{y}^{2}}\right)}{\left(a b-\frac{\kappa_{n}^{2}}{k_{y}^{2}}\right)}
$$

where $c_{s}^{2}=\frac{T_{e}}{m_{i}}$, and $b=\left(1+\lambda_{D e}^{2} k_{y}^{2}\right)$. We have to use Poisson equation to obtain a quadratic equation in $\omega$ while (2) implies $n_{e 1} \simeq n_{i 1}$. The equation (25) is the same as equation (21) of Ref. [1] where two small terms in the denominator are missing. Note that $\lambda_{D e}^{2}<\lambda_{e}^{2}$ and if quasi neutrality is used due to Ampere's law, then (25) yields the basic electrostatic IAW dispersion relation $\omega^{2}=c_{s}^{2} k_{y}^{2}$. If displacement current is retained and Poisson equation is used without using $\omega^{2}, \omega_{p i}^{2}<<\omega_{p e}^{2}$, then one obtains a dispersion relation of coupled three waves; ion acoustic wave, electron plasma wave and high frequency transverse wave [3]. Actually the contribution of displacement current in the curl of Maxwell's equa- 
tion has been neglected for $\omega^{2}<<\omega_{p e}^{2}, c^{2} k^{2}$. This should not mean that the electrostatic part of current is also divergence free, in our opinion. In the divergence part of Maxwell's equation $\omega^{2}<<\omega_{p e}^{2}$ is used but $v_{t e}^{2} k_{y}^{2}$ term is assumed to be important. It may be mentioned that in this treatment, Ampere's law does not imply quasi-neutrality necessarily. We need a coupling of divergence part and cure part of the current and for this we need to assume $1<\lambda_{D e}^{2} k_{y}^{2}$ in the limit $1<\omega^{2}<<\omega_{p e}^{2}, c^{2} k^{2}$.

\section{Unstable Electromagnetic Wave}

Now we present a simple but interesting picture of ideal plasma assuming ions to be cold. The divergence and curl of (1) give, respectively,

$$
\partial_{t} \nabla \cdot\left(n_{0} \mathbf{v}_{e 1}\right)=-\frac{e}{m_{e}} n_{0} \nabla \cdot \mathbf{E}_{1}-\frac{e}{m_{e}} \nabla n_{0} \cdot \mathbf{E}_{1}-\frac{1}{m_{e}}\left(\nabla \cdot \nabla p_{e 1}\right)
$$

and

$$
\partial_{t}\left(\nabla \times \mathbf{v}_{e 1}\right)+\left(\kappa_{n} \times \partial_{t} \mathbf{v}_{e 1}\right)=-\frac{e}{m_{e}} \kappa_{n} \times \mathbf{E}_{1}-\frac{e}{m_{e}} \nabla \times \mathbf{E}_{1}
$$

where $\kappa_{n}=\left|\frac{1}{n_{0}} \frac{d n_{0}}{d x}\right|$ and $\nabla n_{0}=+\hat{\mathbf{x}}\left|\frac{d n_{0}}{d x}\right|$ has been assumed. If initially electric field was purely electrostatic i.e. $\mathbf{E}_{1}=-\nabla \varphi_{1}$, then it will develop a rotating part as well if $\nabla n_{0} \times \mathbf{E}_{1} \neq 0$, as is indicated by the right hand side (RHS) of (27).

Therefore, in an inhomogeneous plasma electron thermal fluctuations can take place due to low frequency perturbations of electric field which has both transverse and longitudinal components. Then these thermal fluctuations can couple with IAW even in the quasi-neutrality limit. 
However, we use the Poisson equation

$$
\nabla \cdot \mathbf{E}_{1}=4 \pi n_{0} e\left(\frac{n_{i 1}}{n_{0}}-\frac{n_{e 1}}{n_{0}}\right)
$$

Using (16) and (23), the above equation can be written as,

$$
\begin{gathered}
{\left[\left(L_{0}^{2} W_{0}^{2}\right) \omega^{2}-\left(L_{0}^{2} \omega_{0}^{2}\right) \omega_{p i}^{2}-\omega_{p e}^{2}\left(W_{0}^{2}+v_{t e}^{2} k_{y}^{2}\right)\right] i k_{y} E_{1 y}=} \\
{\left[L_{0}^{2} W_{0}^{2} \omega_{p i}^{2}+\omega_{p e}^{2}\left(W_{0}^{2}+\frac{3}{2} \frac{\kappa_{T}}{\kappa_{n}} v_{t e}^{2} k_{y}^{2}\right) \omega^{2}\right] \kappa_{n} E_{1 x}}
\end{gathered}
$$

In the limit $\omega^{2}, \omega_{p i}^{2}<<\omega_{p e}^{2},(29)$ reduces to,

$$
\begin{gathered}
{\left[\left\{-\frac{3}{2} \omega_{p e}^{2}-v_{t e}^{2} k_{y}^{2}\left(\frac{3}{2}+G_{n}\right)\right\} \omega^{4}\right.} \\
+\left\{\frac{3}{2} v_{t e}^{4} k_{y}^{4}\left(g_{T}^{2}-g_{n T}^{2}\right)-\frac{3}{2} \omega_{p e}^{2} v_{t e}^{2} k_{y}^{2} g_{n T}^{2}+\omega_{p i}^{2} v_{t e}^{2} k_{y}^{2}\left(\frac{3}{2}+G_{n}\right)\right\} \omega^{2} \\
\left.-\frac{3}{2} \omega_{p i}^{2} v_{t e}^{4} k_{y}^{4}\left(g_{T}^{2}-g_{n T}^{2}\right)\right] i E_{1 y} \\
=\left[\frac{3}{2} \omega_{p e}^{2} \omega^{4}-\left\{\omega_{p i}^{2} v_{t e}^{2} k_{y}^{2}\left(\frac{3}{2}+G_{n}\right)-\frac{3}{2} \omega_{p e}^{2} v_{t e}^{2} k_{y}^{2} \frac{\kappa_{T}}{\kappa_{n}}+\omega_{p e}^{2} v_{t e}^{2} k_{y}^{2} G_{n}\right\} \omega^{2}\right. \\
\left.+\frac{3}{2} \omega_{p i}^{2} v_{t e}^{4} k_{y}^{4}\left(g_{T}^{2}-g_{n T}^{2}\right)\right]\left(\frac{\kappa_{n}}{k_{y}} E_{1 x}\right)
\end{gathered}
$$

Equations (14) and (30) yield a linear dispersion relation as follows,

$$
\begin{gathered}
\frac{3}{2}\left[a b-\left(\frac{\kappa_{n}}{\kappa_{y}}\right)^{2}+\frac{2}{3} a \lambda_{D e}^{2} k_{y}^{2} G_{n}\right] \omega^{4} \\
+\left[-\frac{3}{2} \lambda_{D e}^{2} k_{y}^{2} v_{t e}^{2} k_{y}^{2}\left(g_{T}^{2}-g_{n T}^{2}\right) a+\frac{3}{2} v_{t e}^{2} k_{y}^{2} g_{n T}^{2} a-c_{s}^{2} k_{y}^{2}\left(\frac{3}{2}+G_{n}\right) a\right. \\
\left.+\left(\frac{\kappa_{n}}{k_{y}}\right)^{2}\left\{c_{s}^{2} k_{y}^{2}\left(\frac{3}{2}+G_{n}\right)+v_{t e}^{2} k_{y}^{2} G_{n}-\frac{3}{2} v_{t e}^{2} k_{y}^{2} \frac{\kappa_{T}}{\kappa_{n}}\right\}\right] \omega^{2}
\end{gathered}
$$




$$
+\frac{3}{2} a c_{s}^{2} k_{y}^{2} v_{t e}^{2} k_{y}^{2}\left(g_{T}^{2}-g_{n T}^{2}\right)-\left(\frac{\kappa_{n}}{k_{y}}\right)^{2} c_{s}^{2} k_{y}^{2} v_{t e}^{2} k_{y}^{2}\left(g_{T}^{2}-g_{n T}^{2}\right)=0
$$

This equation contains a coupling of both the electromagnetic waves described by the dispersion relations (20) and (25). We are interested in finding out a simple dispersion relation in quadratic form to look at the physical phenomena more clearly.

For this we assume $g_{n T}=g_{T}$ i.e. $\kappa_{T}=\kappa_{n}$ and in this case (31) becomes,

$$
\omega^{2}=\frac{2}{3 H_{0}}\left\{c_{s}^{2} k_{y}^{2}\left(\frac{3}{2}+G_{n}\right)\left(a-\kappa_{n}^{2} / k_{y}^{2}\right)-v_{t e}^{2} \kappa_{n}^{2}\left(1+\frac{3}{2} \lambda_{e}^{2} k_{y}^{2}\right)\right\}
$$

where $H_{0}=\left[a b-\frac{\kappa_{n}^{2}}{k_{y}^{2}}+\frac{3}{2} a \lambda_{D e}^{2} k_{y}^{2} G_{n}\right]$. In the quasi-neutrality limit, we have $\mathrm{b}=1$ in the above electromagnetic dispersion relation and it reduces to

$$
\omega^{2} \simeq \frac{2}{3 H_{1}}\left\{c_{s}^{2} k_{y}^{2}\left(\frac{5}{2} a \frac{\kappa_{n}^{2}}{k_{y}^{2}}\left(a+\frac{5}{2}\right)\right)-v_{t e}^{2} \kappa_{n}^{2}\left(1+\frac{3}{2} \lambda_{e}^{2} k_{y}^{2}\right)\right\}
$$

where $H_{1}=\left(a-\kappa_{n}^{2} / k_{y}^{2}\right)$ and for $\kappa_{n}=0$, we obtain $\omega^{2}=\frac{5}{3} c_{s}^{2} k_{y}^{2}$.

It is important to mention that the transverse and longitudinal components of electric field do not decouple in the presence of electron thermal fluctuations even if quasi-neutrality is used. If thermal fluctuations are ignored (32) reduces to (25) and if ion dynamics is neglected we recover the result of equation (20). In principle both the waves described by (20) and (25) have frequencies very near to each other under local approximation in plasmas of low-Z materials. Therefore the coupled mode (32) is a fundamental unstable electromagnetic wave of unmagnetized inhomogeneous plasmas. If $\lambda_{e}^{2} k_{y}^{2}<m_{e} / m_{i}$, then $a \simeq 1$ and $b=1$. In this case Eq. (33) predicts purely growing electrostatic fluctuations for $\frac{m_{e}}{m_{i}}<\left(\frac{\kappa_{n}}{k_{y}}\right)^{2}$ because the second term on RHS of (33) can become larger than the first term. 


\section{Application}

As an illustration we consider the parameters of classical laser-induced plasma as $n_{0} \sim 10^{20} \mathrm{~cm}^{-3}$ and $T_{e}=100 \mathrm{ev}$. Then we obtain $c_{s} \sim 10^{7} \mathrm{~cm} / \mathrm{Sec}, v_{t e}=$ $4.18 \times 10^{8} \mathrm{~cm} / \mathrm{Sec}, \lambda_{e} \sim 0.53 \times 10^{-4} \mathrm{~cm}, \lambda_{D e} \sim 0.74 \times 10^{-6} \mathrm{~cm}$, and $\omega_{p i} \sim$ $1.3 \times 10^{13} \mathrm{rad} / \mathrm{Sec}$. If $\kappa_{n} \sim 10^{4} \mathrm{~cm}^{-1}$ and we choose $k_{y} \sim 10^{5} \mathrm{~cm}^{-1}$, in Hy-

drogen plasma we find $\frac{m_{e}}{m_{i}}<\left(\frac{\kappa_{n}}{k_{y}}\right)^{2}=10^{-2}$. We obtain $\mathrm{a}=29, \mathrm{~b}=1.0055$, $v_{t e} \kappa_{n} \sim 4.18 \times 10^{12} \mathrm{rad} / \mathrm{Sec}$ and $c_{s} k_{y} \sim 10^{12} \mathrm{rad} / \mathrm{Sec}$. Since $\frac{m_{e}}{m_{i}}<\lambda_{D e}^{2} k_{y}^{2}$, we use the result of Poisson equation (32) and obtain

$$
\omega \simeq \pm i\left(5.8 \times 10^{12}\right)
$$

Note that it gives a purely growing electromagnetic instability. Furthermore $c_{s} k_{y} \lesssim|\omega| \lesssim \omega_{p i}$ and hence one should not ignore ion dynamics.

The instability appears due to the second term on RHS which enters because of pure electron dynamics. But fluctuations are at ion time scales. Therefore, ions must be treated to be mobile. The instability can also appear in the quasineutrality limit.

If $\kappa_{n} \sim 10^{3} \mathrm{~cm}^{-1}$ and we choose $k_{y} \sim 10^{4} \mathrm{~cm}^{-1}$, then $\lambda_{D e}^{2} k_{y}^{2}<m_{e} / m_{i}$ and hence we use quasi-neutrality. Then $\mathrm{a}=1.28$ and (33) yields,

$$
\omega \simeq \pm i\left(3.6 \times 10^{12}\right)
$$

Again we see a purely growing electromagnetic mode with $|\omega| \lesssim \omega_{p i}$.

\section{Discussion}

A low frequency unstable electromagnetic wave has been studied in unmagnetized inhomogeneous plasmas. This wave can be an intrinsic source of magnetic 
field fluctuations in initially unmagnetized plasmas. Dynamics of both electrons and ions are important in this instability.

These fluctuations can become unstable when the density and temperature gradients are parallel to each other as is the case, in the stellar cores. Similarly in laser-induced plasmas these gradients can be in the same direction due to external conditions.

In a statistical ensemble of charged particles, the electrostatic fluctuations are quite natural. Any initial electrostatic field perturbation can produce its transverse component as well in the presence of density gradient. This phenomenon can cause a coupling of ion acoustic wave (IAW) with the low frequency transverse magnetostatic mode. This coupled mode has already been investigated more than a decade ago [1]. But it can exist in a relatively shorter wavelength range for $\frac{m_{e}}{m_{i}}<\lambda_{D e}^{2} k^{2}$. In the quasi-neutrality limit, the IAW and magnetostatic modes decouple.

Another interesting mechanism has been proposed for the generation of a transverse wave in as electron plasma. In this case the transverse wave (MEDV mode) is produced by the electron temperature perturbation $[13,14]$. This mode does not have density fluctuations. In the present investigations it has been shown that a low frequency electromagnetic wave seems to appear in a non-uniform unmagnetized electron plasma having both the longitudinal and transverse electric field components. But the frequencies of both the above mentioned modes are of the order of $v_{t e} \kappa_{n}$ or even smaller.

Since frequencies $v_{t e} \kappa_{n}$ and $c_{s} k_{y}$ can be very near to each other, therefore ions should not be assumed as stationary. We need to consider ion dynamics as well.Then we obtain a coupled linear dispersion relation which is fourth order polynomial in $\omega$. If $\kappa_{T}=\kappa_{n}$ is assumed, then it reduces to a quadratic equation (32). It indicates the presence of a partially transverse and partially longitudi- 
nal unstable wave in nonuniform unmagnetized electron-ion plasmas. Dynamics of both electrons and ions take part in the generation of this instability. The electron temperature fluctuations are necessary to produce this wave. It is also important to mention that this wave can exist in the quasi-neutrality limit as well.

In our opinion, this electromagnetic instability plays an important role in the generation of fluctuating magnetic fields in initially unmagnetized plasmas. The nonlinear saturation mechanisms need to be investigated which can contribute to the magnetization of the plasmas. Therefore the electromagnetic waves and instability presented here can be important in the study of magnetic field generation in laser-induced and astrophysical plasmas. 


\section{References}

[1] H. Saleem, Phys. Rev. E 54, 4469 (1996).

[2] C. Chu, M. S. Chu and T. Ohkawa, Phys. Rev. Lett. 41, 653 (1978).

[3] H. Saleem, K. Watanabe and T. Sato, Phys. Rev. E 62, 1155 (2000).

[4] A. Stamper, K. Papadapoulos, R. N. Sudan, S. O. Dean, E. A. McLean, and J. M. Dawson, Phys. Rev. Lett. 26, 1012 (1971).

[5] J. A. Stamper, E. A. McLean and B. H. Ripin, Phys. Rev. Lett. 40, 1177 (1978).

[6] A. Raven, O. Willi, and R. T. Rumsby, Phys. Rev. Lett. 41, 554 (1978).

[7] B. A. Altercop, E. V. Mishin, and A. A. Rukhadze, Pis'ma Zh. Eksp. Teor. Fiz. 19, 291 (1974) [JETP Lett. 19], 170 (1974).

[8] L. A. Bol'shov, Yu, A. Dreizin, and A. M. Dykhne, Pis'ma Zh. Eklsp. Teor. Fiz. 19, 288 (1974) [JETP Lett. 19, 168 (1974)].

[9] K. A. Brueckner and S. Torna, Rev. Mod. Phys. 46, 325 (1974).

[10] G. J. Pert, J. Plasma Phys. 18, 227 (1977).

[11] C. E. Max, W. M. Manheimer, and J. J. Thomson, Phys. Fluids 21, 128 (1978).

[12] M. G. Haines, Phys. Rev. Lett. 47, 917 (1981).

[13] R. D. Jones, Phys. Rev. Lett. 51, 1269 (1983).

[14] M. Y. Yu and Xiao Chijin, Phys. Fluids 30, 3631 (1987).

[15] A. A. Kingssep, K. V. Chukhar, and V. V Yan'Kov, in Reviews of Plasma Physics, edited by B. B. Kadomtsev (Cousultatn Bureau, New York 1990), Vol. 16, p. 243. 
[16] L. A. Bol'shov, A. M, Dykhne, N. G. Kowalski, and A. I. Yudin. in Handbook of Plasma Physics, edited, by M. N. Rosenbluth and R. Z. Sagdeev (Elsevier Science, New York, 1991), Vol. 3, p. 519.

[17] . B. Eliasson and P.K. Shukla, Phys. Rev. Lett. 99, 205005 (2007).

[18] R.L. Stenzel etal. Phys. Rev. Lett. 96, 095004 (2006).

[19] R.L. Stenzel, Plasma Phys. Controlled Fusion 49, A17 (2007). 